\begin{tabular}{|c|l|}
\hline Title & Hysteretic mode exchange in the wake of two circular cylinders in tandem \\
\hline Author(s) & Tasaka, Y uji; Kon, Seiji; Schouveiler, Lionel; Gal, Patrice Le \\
\hline Citation & $\begin{array}{l}\text { Physics of Fluids, 18, 084104 } \\
\text { https://doi.org/10.1063/1.2227045 }\end{array}$ \\
\hline Issue Date & 2006-08 \\
\hline Doc URL & http://hdl.handle.net/2115/14717 \\
\hline Rights & Copyright $\odot 2006$ A merican Institute of Physics \\
\hline Type & article \\
\hline File Information & PF-2006-18.pdf \\
\hline
\end{tabular}

Instructions for use 


\title{
Hysteretic mode exchange in the wake of two circular cylinders in tandem
}

\author{
Yuji Tasaka and Seiji Kon \\ Laboratory for Flow Control, Graduate School of Engineering, Hokkaido University, N-13 W-8, \\ 060-8628 Sapporo, Japan \\ Lionel Schouveiler and Patrice Le Gal \\ Institut de Recherche sur les Phénomènes Hors Equilibre, UMR 6594, CNRS \& Universités d'Aix-Marseille \\ I et II, 49 rue F. Joliot-Curie, BP146, 13384 Marseille, France
}

(Received 14 March 2006; accepted 16 June 2006; published online 23 August 2006)

\begin{abstract}
Our experimental study is devoted to the analysis of the flow past two tandem circular cylinders near the vortex shedding threshold. A recent bidimensional numerical analysis of this flow [Mizushima and Suehiro, Phys. Fluids 17, 104107 (2005)] has predicted that the bifurcation diagram should become complex in the vicinity of the instability threshold. Subcritical and saddle node bifurcations that lead to hysteretic exchanges between two different modes of vortex shedding were detected for particular distances of separation of the cylinders. We present here visualizations and velocity measurements of this flow in a water channel that prove the robustness of the complexity of the bifurcation diagram in real flows. (C) 2006 American Institute of Physics. [DOI: 10.1063/1.2227045]
\end{abstract}

\section{INTRODUCTION}

The interest in flows around arrangements of cylinders goes back many years, and many studies have already revealed complex hysteretic behavior of the flow characteristics, such as the lift or drag coefficients, or the Strouhal number of the vortex shedding from pairs of cylinders. These configurations are of great practical importance as they often occur in flow control strategies, where extra bodies are often used to modify, or to probe, the original wakes. The reviews of Zdrakovich ${ }^{1}$ and Ohya, Okajima, and Hayashi ${ }^{2}$ show that many different flow patterns and wake interferences are determined by the relative position of the cylinders. The situation where the cylinders are aligned to the flow direction (the so-called tandem arrangement) has been of particular interest and a critical spacing (around 3.8 cylinder diameters) has long been detected by Ishigai et al. ${ }^{3}$ This feature has since been confirmed in high Reynolds number flows. ${ }^{4-6}$ To our knowledge, the only low Reynolds number experiments of Tanida et al. ${ }^{7}$ shows a discontinuity in the drag coefficient curve for a spacing around of 3.9 diameters. These results were further confirmed by Ohmi and Imaichi ${ }^{8}$ and by the recent numerical simulations of Meneghini et al. ${ }^{9}$ and Sharman et al. ${ }^{10}$ To our knowledge, the most complete description of the tandem cylinder wake at low Reynolds numbers was recently given by Mizushima and Suehiro. ${ }^{11}$ Using both linear stability analysis and direct numerical simulation, they investigated the instability of the two-dimensional wake of two tandem cylinders, which were arranged as shown in Fig. 1. The control parameters of the problem are the gap ratio $\Gamma$, which is the distance between the cylinder axes $l$ divided by the diameter $d$ of each cylinder and the Reynolds number $\operatorname{Re}=U d / \nu$, where $U$ is the upstream velocity and $\nu$ the kinematic viscosity of the fluid. Their calculations were performed for a range of $\Gamma$ going from 1 to 6 and for Re ranging from 60 to 100. The main results of their study are as follows. At small Reynolds numbers, i.e., for Re close to 60, steady symmetric solutions were obtained independently of $\Gamma$. The separated flow from the upstream cylinder reattached to the downstream one; hence, the two cylinders could be regarded as a unique elongated obstacle. At higher Reynolds numbers, i.e., for Re around 100, the spatial and temporal symmetries were found to break down and oscillations occurred. One of the two vortex shedding regimes was found to occur depending on $\Gamma . \Gamma=2$ and 4 were the two configurations chosen to illustrate the two vortex shedding regimes. It was shown that for $\Gamma=2$, contrary to the case $\Gamma=4$, the flow in the gap between the cylinders has no oscillation, and the oscillations measured downstream of the cylinders are smaller and slower than for $\Gamma=4$. These characteristics illustrated the main differences between the two vortex shedding modes. The direct numerical simulation showed abrupt exchanges between these two stable solutions for $3.1 \leqslant \Gamma$ $\leqslant 3.4$. Complete transition diagrams were explicitly calculated; they exhibited saddle node and subcritical bifurcations when $\operatorname{Re}$ and $\Gamma$ were varied. Additionally, the hysteretic evolution of the Strouhal number and of the lift and drag coefficients associated with the two vortex shedding modes were presented. In the present experimental study, we confirm the existence and their hysteretic exchange of the two vortex shedding regimes past two tandem circular cylinders as observed in the results of the bidimensional numerical calculation of Mizushima and Suehiro. ${ }^{11}$ Flow patterns at each Reynolds number are observed by visualization using fluorescent dye for increasing and decreasing Re, and for different values of $\Gamma$. An ultrasonic velocity profiler (UVP) has also been employed to measure the velocities and their fluctuation profiles in the near wake. As presented later, the different bifurcations are quantitatively described and if the critical parameters are shifted compared to their numerically predicted values, the main sequence of bifurcations is recovered despite finite size effects or eventual three-dimensional (3D) instabilities. We conclude that the scenario predicted by Mi- 


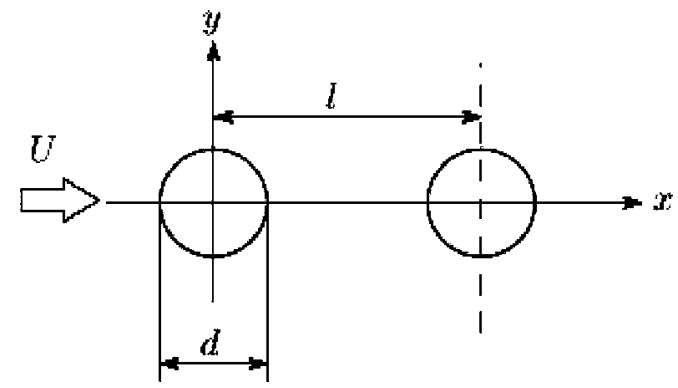

FIG. 1. Coordinates and symbols

zushima and Suehiro is robust and can occur in real or even industrial flows.

\section{EXPERIMENTAL ARRANGEMENT}

Experiments were performed in a free surface water channel with a test section that was $1400 \mathrm{~mm}$ long with a depth of water at rest of $450 \mathrm{~mm}$, and a slightly diverging width to compensate for the downstream growth of the boundary layers on the walls. The width of the section around the cylinders was close to $380 \mathrm{~mm}$. The Reynolds numbers were determined by the measurement of the flow rate in the water channel with an accuracy of 5\%. The three walls are made of glass allowing visualization. Two calibrated stainless steel $4 \mathrm{~mm}$ diam pipes were used as the tandem circular cylinders and were vertically fixed in the channel by acrylic end plates which were drilled to let the cylinders pass through two holes. The plates are aligned with the flow. Different pairs of holes, separated by different distances, were drilled to allow the variation of the distance 1 between the cylinders. The distance between the plates was $110 \mathrm{~mm}$ (see Fig. 2), leading to an effective length of $110 \mathrm{~mm}$ for each cylinder. This experimental arrangement does not allow for end effect control in order to get parallel shedding. ${ }^{12}$ An electrochemical visualization technique that uses the oxidation of a tin wire ${ }^{13}$ showed that the shedding is oblique to the cylinder axes. Note that the effective aspect ratio of the elongated body formed by the pair of cylinders, depends of their spacing and can be as small as 5. This finite size effect is known to affect significantly the values of the different bifurcation thresholds, ${ }^{14,15}$ but not, as we will see, the main succession of bifurcations, whose comparison with

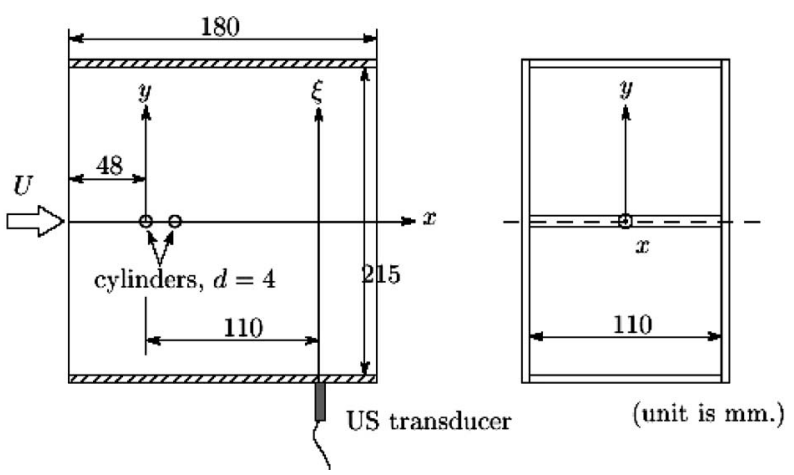

FIG. 2. Top view and front view of the experimental arrangement and scales of the test section. the two-dimensional (2D) numerical simulations is still pertinent. Two other acrylic side plates were also located parallel to the tandem cylinders in order to reduce the disturbance induced by the setting of the ultrasonic transducer in the flow. The Reynolds number Re was varied from 90 to 300, where the minimum Re was determined by the minimum working velocity of the water channel. The gap ratio $\Gamma$ was varied over the range $2.0 \leqslant \Gamma \leqslant 8.0$ with an accuracy better than 0.05 . A fluorescent dye solution of fluorescein disodium salt was gently injected at midheight from a $0.5 \mathrm{~mm}$ hole made at the rear side of the upstream cylinder and was illuminated by an Argon Laser light sheet emitted from the downstream end of the channel. Visualized flow patterns were captured by a CCD camera and recorded on a PC. A UVP was then used to measure the velocity profiles and the velocity fluctuations in the wake. An ultrasonic transducer, with a $5 \mathrm{~mm}$ effective diameter, was located at midheight, $110 \mathrm{~mm}$ downstream of the cylinders, perpendicular to the stream direction. $4 \mathrm{MHz}$ ultrasound bursts were emitted through one side plate. Hydrogen bubbles were generated from a $0.1 \mathrm{~mm}$ diam platinum wire which was located in the front of the upstream cylinder perpendicular to the cylinder. These bubbles (roughly $100 \mu \mathrm{m}$ in size) were used to reflect the ultrasound bursts that propagated through the flow. Note that it was confirmed by visualization that the bubbles did not influence the flow pattern. Ultrasound echo signals captured by the transducer were converted to instantaneous transverse velocity profiles perpendicular to the cylinder $v(\xi, t)$ ( $\xi$ axis defined in Fig. 2) by a UVP monitor model X2 (Met-Flow S.A.). The spatial resolution of this method is $0.74 \mathrm{~mm}$ along the ultrasonic beam, and around $5 \mathrm{~mm}$ in the longitudinal direction and we estimated the accuracy of the velocity measurements around $\pm 3 \mathrm{~mm} / \mathrm{s}$.

\section{RESULTS AND DISCUSSIONS}

\section{A. Visualization of the flow patterns}

Figure 3 shows the visualizations of the flow patterns for different gap ratios $\Gamma$ and different Reynolds numbers Re. It illustrates the three states of the tandem wake in the vicinity of the vortex shedding threshold. Figure 3(a) shows the wake for $\Gamma=4.0$ and $R e=90$. It is stable and stationary and only a very slight oscillation is visible on the figure. For this $\Gamma$, the critical Reynolds number for vortex shedding is slightly above 90 and the dye pattern is symmetric with respect to the $x$ axis. The separated flow from the upstream cylinder reattaches to the downstream cylinder and thus the streamlines are closed in the gap between the cylinders. When increasing the Reynolds number Re, some oscillations appear and become larger when the Reynolds number is further increased. Vortices are formed in the wake downstream of the second cylinder as shown in Fig. 3(b) for $\Gamma=4.0$ and $R e=150$. The streamlines in the gap still close and the cylinders form a single elongated obstacle. For this $\Gamma$, even for higher Reynolds numbers, the closed area between the cylinders never breaks. On the contrary, the visualization of the wake for $\Gamma$ $=5.0$ and $\operatorname{Re}=150$ of Fig. 3(c) shows that the flow in the gap oscillates even for the lowest Reynolds number investigated. 


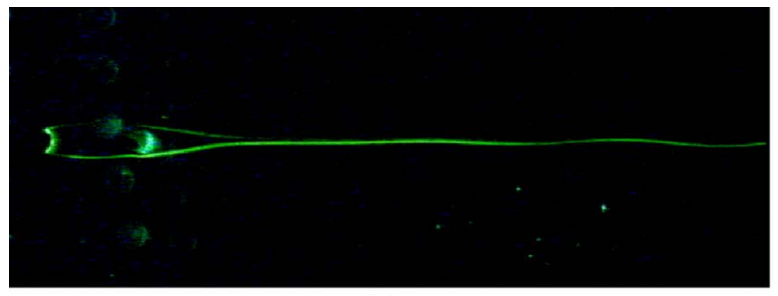

(a)

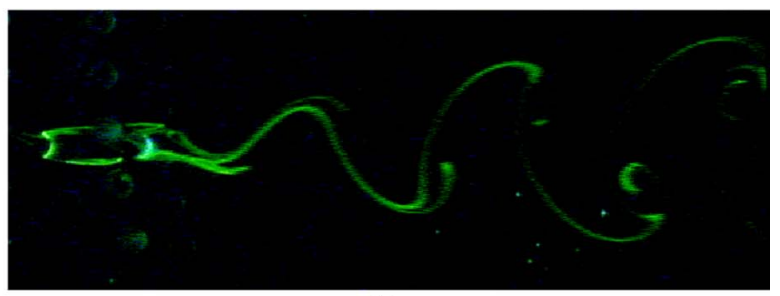

(b)

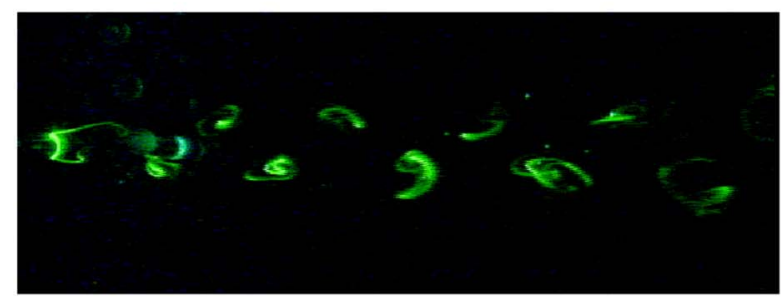

(c)

FIG. 3. (Color online) Visualized flow pattern; (a) stationary stable flow for $\Gamma=4.0$ and $\operatorname{Re}=90$, (b) slow mode of vortex shedding at $\Gamma=4.0$ and $R e=150$, and (c) fast mode of vortex shedding at $\Gamma=5.0$ and $\mathrm{Re}=150$. These visualizations correspond to Figs. 3 and 4 of the numerical simulation of Ref. 11 .

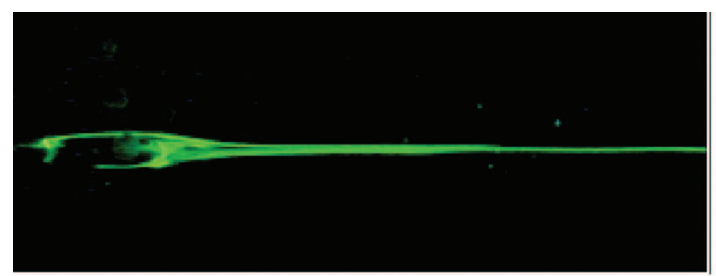

(a) $R e=90$

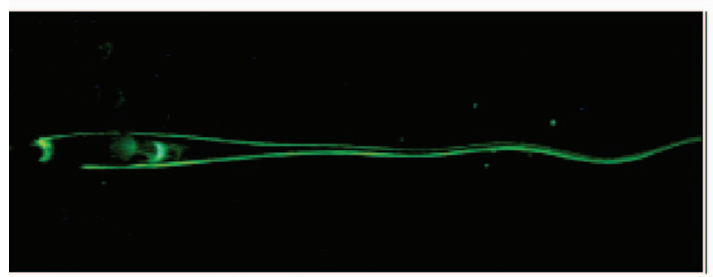

(b) $R e=110$

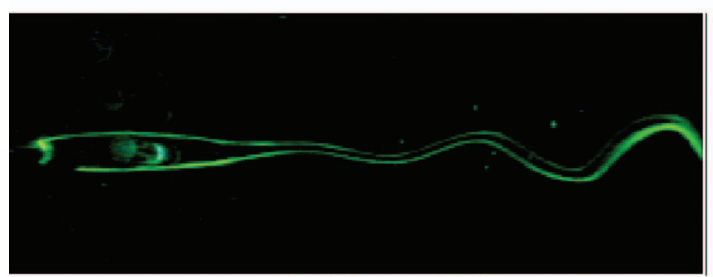

(c) $R e=130$

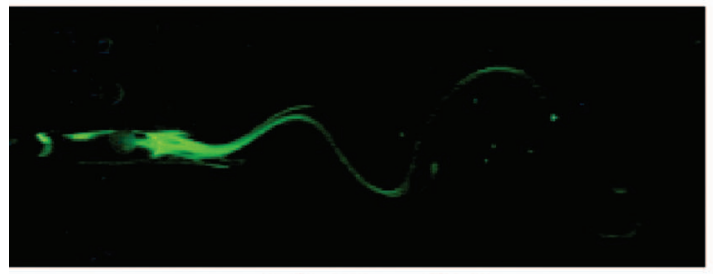

(d) $R e=150$

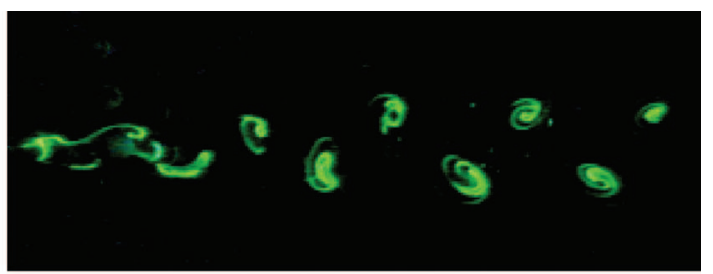

(h) $R e=90$

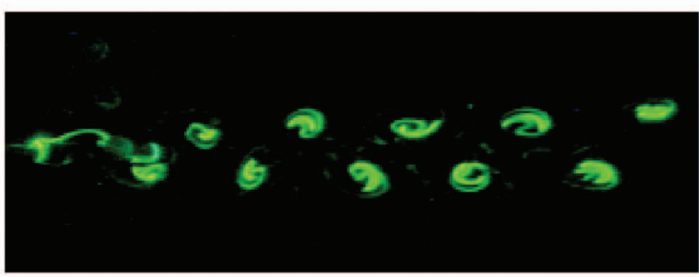

(g) $R e=110$

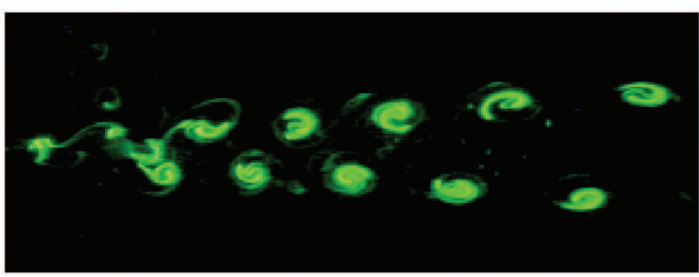

(f) $R e=130$

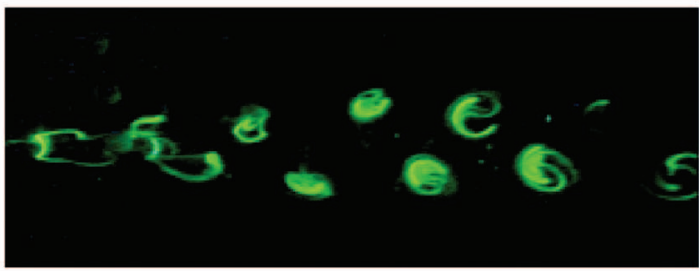

(e) $R e=150$

FIG. 4. (Color) Transition of the flow pattern from oscillating mode to vortex shedding mode at $\Gamma=5.0$, where Reynolds number increases from (a) to (d) and decreases from (e) to (h). 
The vortices are shed from the upstream cylinder and are disturbed by the presence of the downstream cylinder. In particular, we have observed that the vortices frequently merge together and that their positions oscillate in the spanwise direction. We call these two different oscillating modes associated to the vortex shedding respectively the "slow mode" [Fig. 3(b)] and the "fast mode" [Fig. 3(c)]. These modes correspond to the modes that were computed in the numerical study of Mizushima and Suehiro. ${ }^{11}$ The transition between the slow and fast vortex shedding mode is abrupt. The values of Re that this occurs at depends on $\Gamma$. Figure 4 shows a series of visualizations that illustrate the transition of the flow when increasing or decreasing the Reynolds number between 90 and 150 for $\Gamma=5.0$. When increasing the Reynolds number quasistatically from $\mathrm{Re}=90$, the slow mode first bifurcates. The oscillation of the dye streak grows with respect to Re [from Fig. 4(a) to Fig. 4(d)]. This mode then becomes unstable at $\mathrm{Re}=150$ [Fig. 4(d)] and spontaneously changes to the fast vortex shedding mode as shown in Fig. 4(e). Thus, $R e=150$ is a turning point for the mode exchange at $\Gamma=5.0$ and the fast vortex shedding mode exists and is stable for higher Reynolds numbers. When decreasing from $\operatorname{Re}=150$ to 90 , the flow does not return to the slow mode and the fast mode remains for Reynolds numbers much less than 150 [from Fig. 4(e) to Fig. 4(h)]. Performing a series of experiments by incrementing $\Gamma$ in steps of 0.1 , such a hysteretic mode exchange is observed for $4.6 \leqslant \Gamma \leqslant 5.0$. Note that this range of $\Gamma$ is shifted in our experiment in comparison to the values observed in the numerical analysis $^{11}$ where it takes place for $3.05<\Gamma<3.4$. The finite size of the cylinders is certainly at the origin of this discrepancy between the experiment and the 2D simulation. In addition, we have observed that the slow oscillating mode can be easily disturbed and a jump to the fast vortex shedding mode can be triggered by small but finite amplitude perturbations-slight oscillations of the upstream cylinder, for instance-although the current Reynolds number can be smaller than the turning point threshold. However, let us note that this slow oscillation mode is stable to any perturbations for $\Gamma<4.6$.

\section{B. Measurement of the Strouhal number}

The UVP was then used to measure the instantaneous velocity profiles $v(\xi, t)$ along the propagation line of ultrasound bursts, i.e., the transverse $\xi$ axis (see Fig. 2) and for a streamwise position fixed at $x / d=27.5$. Figure 5 shows the spatio-temporal evolution of the oscillation of the transverse velocity profile for $\Gamma=4.6$ and $\operatorname{Re}=120$ where the flow is in the slow vortex shedding mode as shown in Fig. 3(b), i.e., periodic and symmetric to the center of the wake $x / d=0$. This temporal evolution of the profiles expresses the typical form of the Bénard-von Kárman vortex street, i.e., that it is symmetrical to the center of the wake. ${ }^{16,17}$ Figure 6 shows a similar space-time diagram but for $\Gamma=4.6$ and $R e=250$, where the flow is in the fast oscillating mode. Note that a slow modulation of the vortex shedding is also clearly visible. We did not study this modulation in great detail. It could be caused by the interaction of the shedding from the first
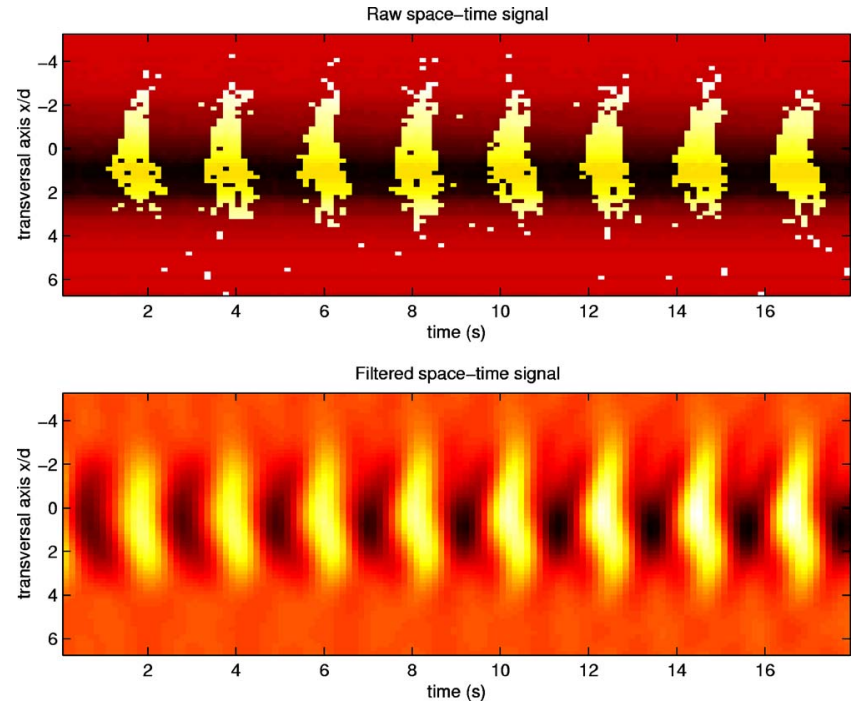

FIG. 5. (Color online) Typical spatio-temporal evolution of the transverse velocity component $v(\xi, t)$ for the slow mode at $\Gamma=4.6$ and $\mathrm{Re}=120$. (a) Raw data from the ultrasound profiler. (b) Low passed data. The amplitude is color (or gray) linearly coded between minimum and maximum.

cylinder with the shedding from the second. As this characteristic was not mentioned in the numerical study of Mizushima and Suehiro, ${ }^{11}$ 3D instabilities can also be evoked to interpret this modulation. Indeed, the concerned range of Reynolds number is larger to the known 3D instability threshold of a single cylinder.

The Fourier power spectrum of the velocity fluctuations have been calculated in order to investigate the variation of the Strouhal number of the vortex shedding with respect to $\operatorname{Re}$ and $\Gamma$. Figure 7 shows the spatially averaged power spectrum of the velocity fluctuation at $\Gamma=4.6$ and $\mathrm{Re}=260$, where the averaging range is for $-10 \leqslant y / d \leqslant 10$. The spectrum presents several peaks with a maximum for a frequency $f_{v}$
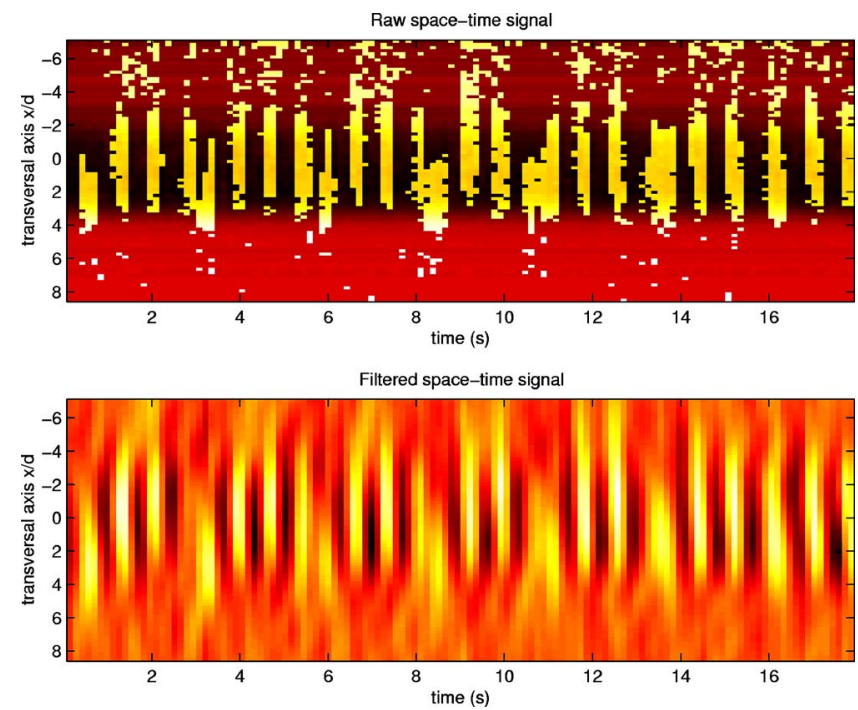

FIG. 6. (Color online) Typical spatio-temporal evolution of the transverse velocity component $v(\xi, t)$ for the fast mode at $\Gamma=4.6$ and $\operatorname{Re}=250$. The slow modulation is clearly visible. (a) Raw data from the ultrasound profiler. (b) Low passed data. The amplitude is color (or gray) linearly coded between minimum and maximum. 


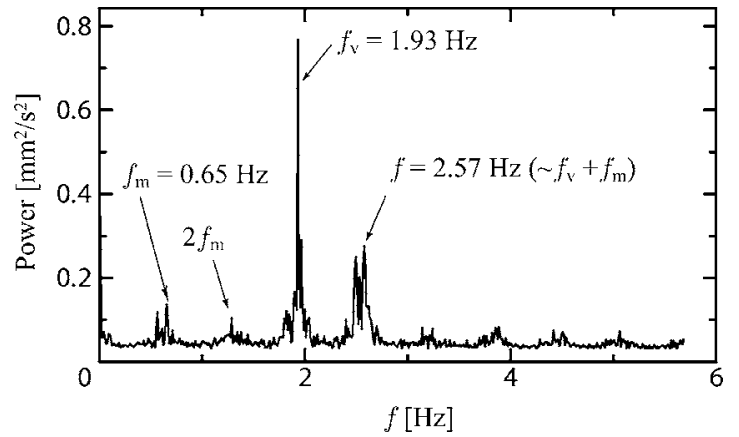

FIG. 7. Spatially averaged power spectrum of the velocity fluctuations $v$ for $\Gamma=4.6$ and $\operatorname{Re}=260$.

$=1.93 \mathrm{~Hz}$, corresponding to the frequency of vortex shedding confirmed from a movie of the visualized flow pattern. A second frequency component in the spectrum at $f_{m}+f_{v}$ $=2.57 \mathrm{~Hz}$ also exists and their interaction creates the slow modulation at a frequency $f_{m}=0.65 \mathrm{~Hz}$ already observed in Fig. 6. Some harmonics of these components are also present in the spectrum. Using these spectra, the Strouhal numbers of the velocity fluctuations can be calculated from the frequency of the maximum peak $f_{v}$ of the spatially averaged power spectrum: $\mathrm{St}=f_{v} d / U$. The accuracy of the measure of St can be estimated to be better than $0.5 \%$.
Figure 8 shows the variation of St with respect to Re for each $\Gamma$ in the range $4.5 \leqslant \Gamma \leqslant 6.0$, where square and cross symbols represent the variation of Re by increasing and decreasing, respectively. At $\Gamma=4.5$, the slow oscillating mode of the flow is fully stable in the range of $\mathrm{Re}$ and thus $\mathrm{St}$ gradually increases with respect to Re from 0.10 to 0.12 [Fig. 8(a)]. At $\Gamma=4.6$ [Fig. 8(b)], the variation of St exhibits the hysteretic mode transition as explained in the preceding section (see Fig. 4). Namely, the variation of St follows two different paths for increasing or decreasing Re. St gradually increases with respect to Re until a turning point corresponding to saddle node bifurcation is met for $\mathrm{Re}_{t 1}=280$. Here, $\mathrm{St}$ experiences an abrupt jump to a higher value around 0.16, which expresses the transition to the fast vortex shedding mode. Due to the hysteresis, this higher value of St can be maintained until $\operatorname{Re}_{t 2}=220$, even if $\operatorname{Re}$ decreases below $\operatorname{Re}_{t 1}$. As already mentioned, for $\operatorname{Re}_{t 2}<\operatorname{Re}<\operatorname{Re}_{t 1}$, the slow oscillating mode, which has a smaller Strouhal number St, is unstable to finite amplitude perturbations and can be easily changed to the fast vortex shedding mode by applying an external forcing on the flow, for instance. On the contrary, for $\operatorname{Re}<\operatorname{Re}_{t 2}$, the slow mode is stable for any perturbations. Such hysteretic mode exchanges can be seen over the entire range $4.6 \leqslant \Gamma \leqslant 5.0$ [see Figs. 8(b)-8(e)]. At $\Gamma=4.7$ [Fig. $8(\mathrm{c})]$, there are also two turning points in the Strouhal num-

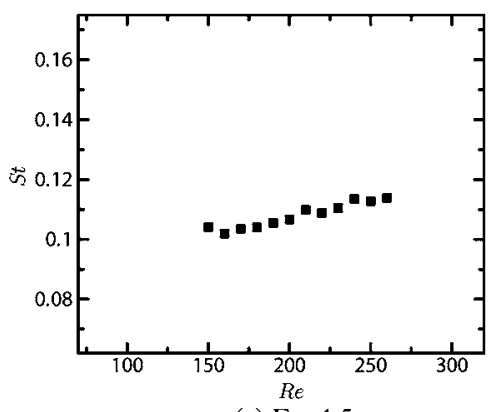

(a) $\Gamma=4.5$

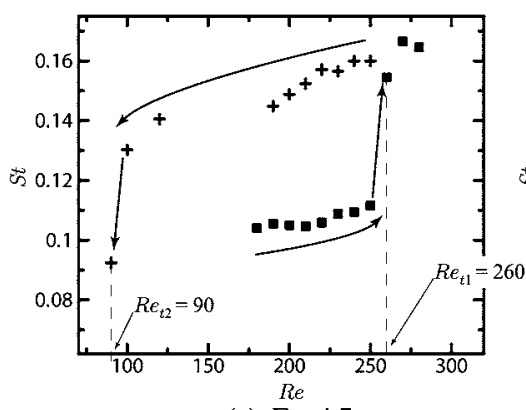

(c) $\Gamma=4.7$

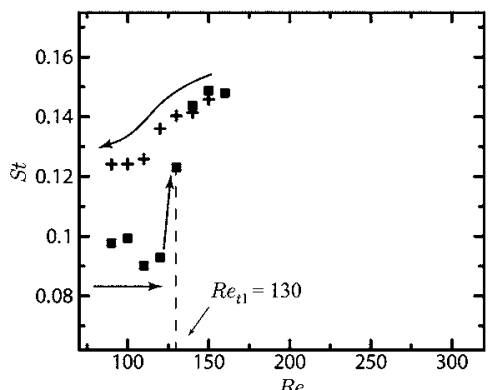

(e) $\stackrel{R e}{\Gamma}=5.0$

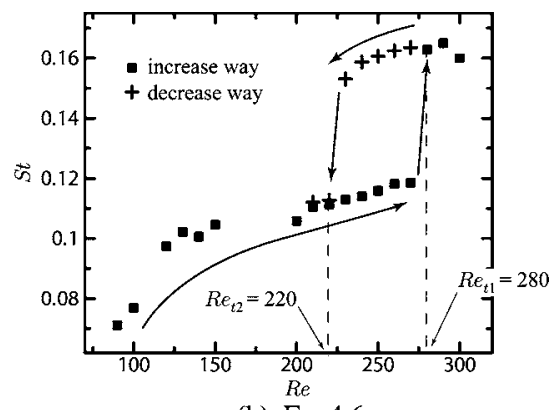

(b) $\Gamma=4.6$

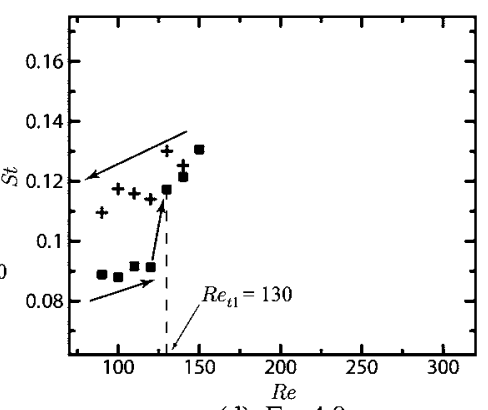

(d) $\Gamma=4.8$

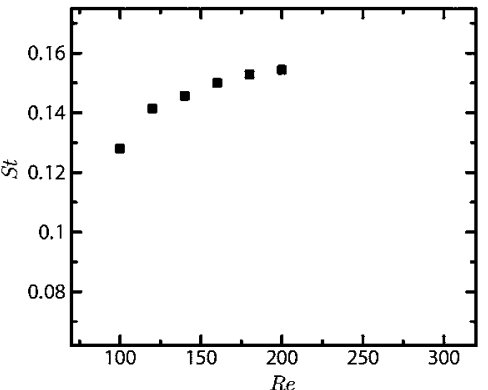

(f) $\stackrel{R e}{\Gamma}=6.0$
FIG. 8. Variation of the Strouhal number St with respect to the Reynolds number Re for each gap ratio $\Gamma$, where square and cross symbols represent, respectively, the variations in the increasing way of Re and in the decreasing way. $\mathrm{Re}_{t}$ represents turning points of the variation. 


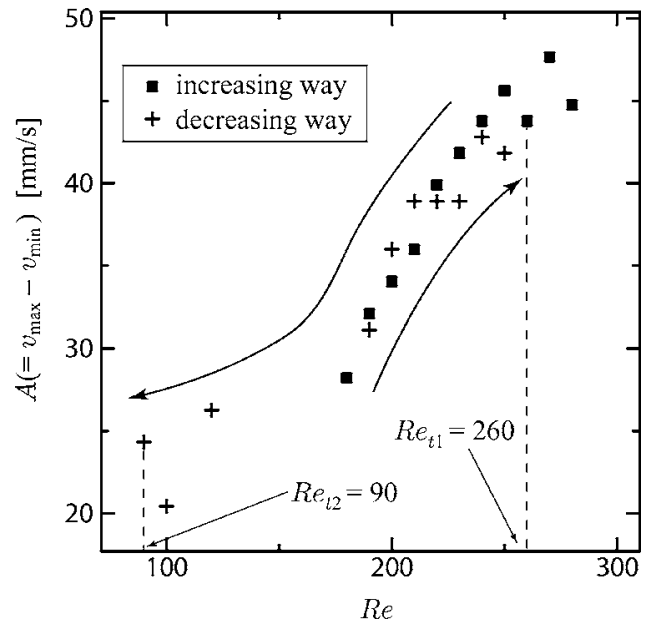

FIG. 9. Variation of the amplitude of velocity fluctuation with respect to Re at $\Gamma=4.7$, where square and cross symbols represent, respectively, the variations in the increasing way of $\mathrm{Re}$ and in the decreasing way. $\mathrm{Re}_{t 1}$ and $\mathrm{Re}_{t 2}$ represent the turning points obtained in Fig. 8(b).

ber evolution. However, in this case, the Reynolds numbers of both turning points $\left(\operatorname{Re}_{t 1}=260\right.$ and $\left.\operatorname{Re}_{t 2}=90\right)$ are smaller than the transition Reynolds numbers observed previously at $\Gamma=4.6$. For $\Gamma \geqslant 4.8$, only one turning point can be observed as we cannot decrease the Reynolds number lower than 90 (because of water channel limitation). Thus we cannot confirm, but can only speculate on the existence of the second turning point $\mathrm{Re}_{t 2}$ for this range of $\Gamma$ [Figs. 8(d) and 8(e)] at Reynolds number lower than 90 . Finally, for $\Gamma=6.0$, St follows continuously the curves corresponding to the fast mode of vortex shedding [Fig. 8(f)]. Note that the Strouhal numbers measured in this experiment are in a range $0.10 \leqslant \mathrm{St}$ $\leqslant 0.16$, which is fully consistent with the results of the numerical work of Mizushima and Suehiro. ${ }^{11}$

\section{Amplitude of velocity fluctuations}

In the results of the numerical calculation of Ref. 11, the amplitude of the velocity fluctuation evolution with respect to the Reynolds number abruptly changes due to the mode transition. Therefore, the subsequent bifurcations can be easily followed. We also investigate the variation of the amplitude in order to clarify if there is also such an abrupt jump in the experiment. The variation of the amplitude is estimated as the variation of the difference between the maximum and the minimum value, $A=v_{\max }-v_{\min }$, in the temporal velocity fluctuation at the center of the wake, $y / d=0$. Figure 9 shows the variation of $A$ for $\Gamma=4.7$, where square and cross symbols represent, respectively, the variation induced by increasing and decreasing Re. As can be seen on the figure, there is no observable typical difference between increasing and decreasing paths of $\mathrm{Re}$, although the flow mode certainly changes at $\mathrm{Re}=260$ [see Fig. 8(c)]. This effect that was perfectly observed in the simulation could come from the low modulation of the velocity field that exists for the fast mode or from the lack of accuracy of the amplitude of the velocity oscillation measurements.

\section{CONCLUSION}

We have confirmed experimentally the existence of two modes (slow and fast modes) of vortex shedding in the flow past two tandem circular cylinders. Our result globally confirm the bidimensional numerical predictions of Mizushima and Suehiro ${ }^{11}$ that resists to finite size effects or eventual 3D instabilities. Abrupt mode transitions when varying the Reynolds number were observed in the flow visualizations and their associated bifurcations were clearly identified through the variation of the Strouhal number. As a consequence of this complex bifurcation diagram, it appears that the mode selection between the slow and the fast vortex shedding is hysteretic. We have also observed that the ranges and the transition values for Reynolds numbers and gap ratios between the cylinders are slightly different from those of the numerical results. This difference is certainly caused by the finite length of the cylinders.

${ }^{1}$ M. M. Zdravkovich, "Review of flow interference between two circular cylinders in various arrangement," J. Fluids Eng. 99, 618 (1977).

${ }^{2}$ Y. Ohya, A. Okajima, and M. Hayashi, "Wake interference and vortex shedding," in Encyclopedia of Fluid Mechanics, edited by N. P. Cheremisinoff (Gulf, Houston, 1988).

${ }^{3}$ S. Ishigai, E. Nishikawa, K. Nishimura, and K. Cho, "Experimental study on structure of gas flow in tube banks with tube axes normal to flow," Bull. JSME 15, 949 (1972).

${ }^{4}$ A. Okajima, "Flows around two tandem circular cylinders at very high Reynolds number," Bull. JSME 22, 504 (1979).

${ }^{5}$ M. Arie, M. Kiya, M. Moriya, and H. Mori, "Pressure fluctuations of the surface of two circular cylinders in tandem arrangement," J. Fluids Eng. 105, 161 (1983).

${ }^{6} \mathrm{G}$. Xu and Y. Zhou, "Strouhal numbers in the wake of two inline cylinders," Exp. Fluids 37, 248 (2004).

${ }^{7}$ Y. Tanida, A. Okajima, and Y. Watanabe, "Stability of circular cylinder oscillating in uniform or in wake," J. Fluid Mech. 61, 769 (1973).

${ }^{8} \mathrm{~K}$. Ohmi and K. Imaichi, "Vortex wake visualization of two circular cylinders," in Flow Visualization IV: Proceedings of the 6th International Symposium, Yokohama, Japan, 5-9 October 1992, edited by Y. Tanida and H. Miyashiro (Springer, Berlin, 1992), pp. 322-326.

${ }^{9}$ J. R. Meneghini, F. Saltara, C. L. R. Siqueira, and J. A. Ferrari, Jr., "Numerical simulation of flow interference between two circular cylinders in tandem and side-by-side arrangements," J. Fluids Struct. 15, 327 (2001).

${ }^{10}$ B. Sharman, F. S. Lien, L. Davidson, and C. Norberg, "Numerical predictions of low Reynolds number flows over two tandem circular cylinders," Int. J. Numer. Methods Fluids 47, 423 (2005).

${ }^{11}$ J. Mizushima and N. Suehiro, "Instability and transition of flow past two tandem circular cylinders," Phys. Fluids 17, 104107 (2005).

${ }^{12} \mathrm{C}$. H. K. Williamson, "Oblique and parallel modes of vortex shedding in the wake of a circular cylinder at low Reynolds numbers," J. Fluid Mech. 206, 579 (1989).

${ }^{13}$ P. Le Gal, I. Peschard, M.-P. Chauve, and Y. Takeda, "Collective behavior of wakes downstream a row of cylinders," Phys. Fluids 8, 2097 (1996).

${ }^{14} \mathrm{~T}$. Lee and R. Budwig, "A study of the effect of aspect ratio on vortex shedding behind circular cylinders," Phys. Fluids A 3, 309 (1991).

${ }^{15}$ P. Albarede and P. A. Monkewitz, "A model for the formation of oblique shedding patterns and chevrons in cylinder wakes," Phys. Fluids A 4, 744 (1992).

${ }^{16}$ J. Dusek, "Spatial structure of the Bénard von-Karman instability," Eur. J. Mech. B/Fluids 15, 619 (1996).

${ }^{17}$ I. Peschard, P. Le Gal, and Y. Takeda, "On the spatio-temporal structure of cylinder wakes," Exp. Fluids 26, 12 (1999). 